\title{
VARIACIÓN LÉXICA Y ZONAS DIALECTALES DE CASTILLA-LA MANCHA ${ }^{1}$
}

\author{
NATIVIDAD HERNÁNDEZ MUÑOZ \\ Universidad de Salamanca
}

\section{INTRODUCCIÓN: VARIACIÓN LÉXICA Y LÉXICO DISPONIBLE}

El estudio de la variación léxica dentro de una comunidad linguística admite diversas posibilidades metodológicas a la hora de recoger y analizar los materiales. En las últimas décadas se ha desarrollado con gran fuerza en el mundo hispánico la línea de investigación de la disponibilidad léxica, que elabora, a través de pruebas de producción escrita, recuentos léxicos con las palabras más disponibles, aquellas que más fácilmente vienen a la mente de los hablantes en torno a un tema o categoría semántica ${ }^{2}$. A la sombra del Proyecto Panhispánico, coordinado por Humberto López Morales, han surgido diversas obras que analizan los léxicos disponibles de comunidades de habla más o menos extensas ${ }^{3}$.

En diversas ocasiones se ha expuesto que los estudios de variación léxica en el campo de la sociolingüística española son relativamente limitados debido

\footnotetext{
1 Este artículo se inscribe en los proyectos del Ministerio de Ciencia y Tecnología HUM2005-00654/FILO y de la Junta de Castilla y León SA 120A07.

${ }^{2}$ Estos recuentos reciben en nombre de diccionarios de léxico disponible o vocabularios disponibles. Se elaboran a través de encuestas realizadas a una muestra representativa de una comunidad linguística. En el Proyecto Panhispánico los informantes preuniversitarios escriben durante dos minutos todas las palabras que conozcan relacionadas con dieciséis campos semánticos (Partes del cuerpo, Profesiones...). Después, por medio de una fórmula matemática que combina el número de veces que aparece cada palabra y la posición que ocupa, se calcula el índice de disponibilidad de cada unidad y se presentan los resultados ordenados por temas, desde la palabra más disponible a la menos disponible.

3 Humberto López Morales, Léxico disponible de Puerto Rico, Madrid, Arco/Libros, 1999. José Antonio Samper Padilla, Juan José Bellón y Marta Samper Hernández, «El proyecto de estudio de la disponibilidad léxica en español», Pautas y pistas en el análisis del léxico hispano (americano), Ávila et al., eds., Frankfurt-Madrid, Vervuert Iberoamericana, 2003, págs. 20-27. José Antonio Samper Padilla, y Marta Samper Hernández, «Aportaciones recientes de los estudios de disponibilidad léxica», LynX Panorámica de Estudios Lingüísticos, 5, 2006, págs. 5-95.
} 
principalmente a dificultades metodológicas ${ }^{4}$. Una de las aplicaciones más productivas de los léxicos disponibles ha sido y es el estudio de la variación social: el análisis de la producción exclusiva de determinadas palabras por los grupos sociales y de la variación de la disponibilidad de las unidades léxicas dependiendo del colectivo que las actualice. Este tipo de encuestas presenta diversos problemas a la hora de validar el uso real de las palabras obtenidas ${ }^{5}$, pero no muy diferentes a las dificultades que conllevan los métodos sociolingüísticos tradicionales en la recolección de materiales léxicos (cuestionarios y encuestas).

Aunque la mayor parte de los estudios sobre léxico disponible se han orientado hacia el análisis de la variación social, también se ha destacado su posible aplicación a la investigación dialectal. Borrego Nieto y Fernández Juncal ${ }^{6}$, en un trabajo realizado en Cantabria, proponían que las herramientas metodológicas de esta línea de investigación podrían servir para predecir el grado de dialectalidad de una comunidad y para identificar de forma rápida las zonas dialectales en un territorio. Esta doble aplicación, diastrática y diatópica ${ }^{7}$, coincide con las tendencias más actuales en los estudios de dialectología y sociolinguística. Para Moreno Fernández ${ }^{8}$ las necesidades de la investigación lingüística moderna han roto las barreras entre estas disciplinas, tanto en los materiales utilizados, como en las diferentes metodologías. Al amparo de esta «dialectología social» nace el Atlas Lingüístico (y etnográfico) de Castilla-La Mancha (ALeCMan), que, según sus autores, es el primer atlas lingüístico español concebido con una inclinación claramente sociolingüística ${ }^{9}$ y cuyas aportaciones serán relevantes en las páginas que siguen.

En resumen, dentro de los estudios de léxico disponible conviven, entre otras, una aplicación sociolingüística y otra de carácter dialectal menos desarro-

\footnotetext{
${ }^{4}$ Julio Borrego Nieto, «Dificultades en el estudio sociolingüístico del léxico», II Encuentro de Lingüistas y filólogos de España y México, Alonso et al., eds., Salamanca, Universidad de Salamanca y Junta de Castilla y León, 1994, págs. 119-131. José Antonio Samper Padilla, «La investigación sociolingüística en España durante los últimos 25 años», Lingüística Española Actual, 26, 2, 2004, págs. 125-149.

${ }^{5}$ Natividad Hernández Muñoz, Hacia una teoría cognitiva integrada de la disponibilidad léxica: el léxico disponible de los estudiantes castellano-manchegos, Colección VITOR-tesis doctorales, Salamanca, Ediciones Universidad de Salamanca, 2006, págs. 13-19.

6 Julio Borrego Nieto y Carmen Fernández Juncal, «Léxico disponible: aplicaciones a los estudios dialectales», IV Congreso de Lingüística General, M. ${ }^{a}$ Dolores Muñoz Núñez et al., eds., Cádiz, Universidad de Cádiz y Universidad de Alcalá, 2002, págs. 297-306.

${ }^{7}$ Se manifiesta aquí tanto el aparato teórico como la terminología desarrollada por Eugenio Coseriu («Los conceptos de 'dialecto', 'nivel' y 'estilo de lengua' y el sentido propio de la dialectología», Lingüística Española Actual, 3, 1981, págs. 1-32).

${ }^{8}$ Francisco Moreno Fernández, «Los estudios dialectales sobre el español de España (19792004)», Lingüística Española Actual, 26, 2, 2004, págs. 65-100.

${ }^{9}$ Pilar García Mouton y Francisco Moreno Fernández, «Sociolingüística en el Atlas Lingüístico (y etnográfico) de Castilla-La Mancha», Actas del I Congreso Anglo-hispano, Madrid, Castalia, 1993, págs. 139-149.
} 
llada. Entre los posibles condicionantes de ese menor desarrollo destaca el hecho de que este tipo de compilaciones favorece la aparición de un léxico estándar y de cierto carácter formal. Sin embargo, en estos materiales también se identifican voces de carácter regional que permiten establecer comparaciones intrarregionales e interregionales (a través tanto de las formas propiamente dialectales, como de la distribución o la variación de la disponibilidad en los términos de uso general). Aunque han sido diversas y fructíferas las investigaciones que han abordado el estudio de los dialectalismos dentro de los vocabularios disponibles ${ }^{10}$, hasta este momento ninguna había desarrollado en conjunto las propuestas de Borrego Nieto y Fernández Juncal ${ }^{11}$ para intentar, con este instrumento metodológico, evaluar el grado y la extensión de las variedades léxicas regionales dentro de un espacio territorial amplio. Castilla-La Mancha ofrece el marco propicio para desarrollar estas aplicaciones, debido a que en ella se produce un cruce singular entre las diferentes hablas peninsulares.

Habida cuenta de la herramienta metodológica de la disponibilidad en la que las variables que definen los grupos de sujetos son determinadas a priori por los investigadores, se necesita un modelo de división dialectal de la comunidad castellano-manchega que pueda ser trasladado a los datos del léxico disponible. Para ello, se ha tomado como punto de partida la propuesta de Moreno Fernández ${ }^{12}$, en su estudio clásico de referencia sobre Castilla La Nueva y los documentos relacionados con el ALeCMan ${ }^{13}$.

\section{LA VARIEDAD LÉXICA CASTELLANO-MANCHEGA}

Tradicionalmente se considera que en Castilla-La Mancha se habla una variedad de español común a los dos tercios septentrionales de España y en especial al habla del centro peninsular y de las dos castillas. En el Manual de Dia-

10 No es aquí el lugar para revisar todas ellas, digamos tan solo que se han investigado los materiales dialectales de los léxicos disponibles de Aragón, Huelva, Cádiz, Málaga, Gran Canaria y Castilla-La Mancha.

11 Julio Borrego Nieto y Carmen Fernández Juncal, «Léxico...», op. cit. Cuando el presente artículo estaba en prensa ha llegado a mis manos el trabajo de M. ${ }^{a}$ Luisa Arnal Purroy, "Áreas lingüísticas y dialectalismos en los jóvenes aragoneses (materiales de disponibilidad léxica en Aragón)», en prensa, estudio que también relaciona los resultados del léxico disponible con las áreas dialectales de Aragón (enfoque semejante al que se adopta en el apartado 4 de este artículo). Ambos trabajos se desarrollaron simultáneamente de forma paralela, hecho que demuestra la novedad y validez del enfoque.

12 Francisco Moreno Fernández, «Castilla La Nueva», Manual de Dialectología Hispánica. El español de España, Manuel Alvar, ed., Barcelona, Ariel Lingüística, 1996, págs. 213-232.

13 Pilar García Mouton y Francisco Moreno Fernández, dirs., Atlas Lingüístico (y etnográfico) de Castilla - La Mancha, http://www.uah.es/otrosweb/alecman, 2003. 
lectología Hispánica de Manuel Alvar ${ }^{14}$ se dedica por primera vez un apartado al castellano hablado en la antigua Castilla La Nueva (territorio del que nace la actual Castilla-La Mancha), diferenciado de las variedades castellanas de Castilla La Vieja, Cantabria y Andalucía. Al habla castellano-manchega se le atribuye un cierto carácter innovador frente al castellano norteño, con un marcado carácter de transición y de frontera por la confluencia de las hablas aragonesas, valencianas, murcianas, andaluzas y extremeñas ${ }^{15}$. Estamos, pues, ante una zona de transición lingǘstica atravesada por gran parte de las isoglosas que delimitan las diferentes variedades castellanas ${ }^{16}$; hecho que facilita la aparición de hablas locales o comarcales, pero dificulta la identificación de una variedad castellanomanchega común (que quizá no exista como tal, ya que las unidades lingüísticas y geográficas no tienen por qué coincidir). Prueba de ello es la escasez de trabajos generales sobre la comunidad autónoma y la abundancia de obras sobre hablas locales de comarcas o municipios más reducidos. García Mouton y Moreno Fernández describen así la realidad dialectal de la comunidad:

Las tierras que estudiaría el ALeCMan cuentan con trabajos restringidos a ámbitos concretos, pero no de tipo general, quizá porque sus variedades lingǘsticas no se consideran dentro de los «dialectos» tradicionales y tienden a verse como un castellano avulgarado, relativamente cercano al normativo. Pero se trata de una región importante para la caracterización dialectal, porque en ella se da el cruce de influencias distintas ${ }^{17}$.

En concreto, las peculiaridades del nivel léxico permiten dividir la comunidad de formas diversas. Por un lado, una clara isoglosa de norte a sur une a las provincias de Ciudad Real y Toledo con una presencia significativa de leonesismos y occidentalismos que marcan la transición hacia los ámbitos extremeños y en algunos casos, andaluces; Guadalajara, Cuenca y Albacete forman una franja oriental que comparte la influencia de aragonesismos y catalanismos y que en algunas ocasiones se prolonga hasta Andalucía oriental; Guadalajara muestra semejanza con las tierras del sur Soria y el sureste de Segovia, mientras que en el sur comparte los rasgos conquenses; Cuenca es tierra de choque y de encuentro entre influencias centrales, orientales y sureñas; finalmente, po-

\footnotetext{
${ }^{14}$ Manuel Alvar, Manual de Dialectología Hispánica, op. cit..

${ }^{15}$ Francisco Moreno Fernández, «Castilla...», op. cit., pág. 214 y Pilar García Mouton, Lenguas y dialectos de España, Madrid, Arco/Libros, 1996, págs. 28-30.

${ }^{16}$ Un ejemplo de ello es la isoglosa de las diferentes realizaciones de la -s implosiva y final de palabra que atraviesa la región en su eje central (Francisco Moreno Fernández, «Geografía lingüística y variacionismo», Contribuciones al estudio de la Lingüística Hispánica, 2, M. Almeida y J. Dorta, eds., Barcelona, Literatura y Ciencia, 1997, págs. 347-356).

17 Pilar García Mouton y Francisco Moreno Fernández, «Proyecto del Atlas Lingüístico (y etnográfico) de Castilla-La Mancha», Actas del I Congreso Internacional de Historia de la Lengua Española, Madrid, Arco/Libros, 1988, págs. 1461-1480.
} 
demos identificar un conjunto homogéneo en la comarca central de La Mancha que comparten las provincias de Toledo, Cuenca, Albacete y Ciudad Real ${ }^{18}$. Esta situación se manifiesta claramente en el léxico disponible de la región, donde conviven términos que pertenecen a variedades aragonesas como ababol, a variedades murcianas como leja o a variedades extremeñas y andaluzas como pileta o tarama.

\section{GEOLINGÜÍSTICA Y LÉXICO DISPONIBLE}

\subsection{Premisas metodológicas}

En el apartado central de esta contribución, voy a contrastar los datos del diccionario de léxico disponible de Castilla-La Mancha ${ }^{19}$ y los materiales léxicos presentados en los mapas del ALeCMan. Antes de comenzar, he de matizar las similitudes y las diferencias entre ambas investigaciones, ya que a pesar de que los materiales son semejantes en los aspectos que se verán más adelante, el punto de partida epistemológico y metodológico se sitúa en planos diferentes.

El ALeCMan nace dentro de la disciplina geolingüística con el objetivo de reflejar los límites y la vitalidad de las variedades diatópicas; sin embargo, posee una clara vocación sociolingüística, factor que lo diferencia de otros atlas regionales peninsulares (vid. §1). El proyecto fue presentado en 1987 (año en el que también se iniciaron las campañas de recogida de la información, completadas en los tres siguientes) y a partir de 2003 los resultados comenzaron a aparecer en la página electrónica del atlas. En el ALeCMan se recogen materiales fonéticos, morfológicos, sintácticos y léxicos mediante cuestionarios y grabaciones. Las encuestas se realizaron en 161 municipios seleccionados a partir de las áreas de dominación socioeconómica. De acuerdo con su enfoque social, en cada municipio se encuestó a un hombre y a una mujer ${ }^{20}$ de entre 55 y 65 años y, para ampliar el espectro diastrático y diafásico, en siete ciudades se entrevistó a ocho sujetos de diferentes edades, sexo, nivel de instrucción y barrio, en tres registros diferentes: formalidad máxima, media y mínima ${ }^{21}$.

El alcance del léxico disponible de Castilla-La Mancha es más específico, ya que solo recoge información de carácter léxico: contabiliza y ordena las pa-

18 Pilar García Mouton y Francisco Moreno Fernández, «Proyecto...», op. cit.; Francisco Moreno Fernández, «Castilla...», op. cit., y Pilar García Mouton, Lenguas..., op. cit.

19 Natividad Hernández Muñoz, Hacia..., op. cit.

${ }^{20}$ El cuestionario de léxico se fragmentó, destinando a la mujer las actividades de ámbito doméstico y el mundo de las creencias y al hombre las actividades agrícolas y ganaderas.

21 Se puede encontrar más información sobre la metodología empleada en Pilar García Mouton y Francisco Moreno Fernández («Proyecto...», op. cit.) y Pilar García Mouton y Francisco Moreno Fernández («Sociolingüística...», op. cit.). 
labras dadas por 680 estudiantes preuniversitarios de toda la región a 16 categorías semánticas. La recogida de información se llevó a cabo entre los años 2000-2003. El perfil social de los sujetos encuestados es más complejo: además del sexo y el nivel sociocultural, incluye variables independientes de carácter específico como el tipo de centro educativo (público-privado) y la localidad de residencia (urbana-rural). Una de las diferencias más significativas entre ambas investigaciones es la edad de los sujetos, ya que mientras en el ALeCMan se entrevista a adultos de edad avanzada, en el léxico disponible participan jóvenes preuniversitarios que no han podido sufrir procesos de interferencias léxicas debidas al desarrollo profesional.

No todos los lugares donde se llevó a cabo la prueba de disponibilidad (30 centros educativos en 24 localidades, véase mapa 2) pertenecen a alguno de los 161 puntos de la encuesta del ALeCMan ${ }^{22}$, principalmente porque las encuestas de léxico disponible solo se pueden llevar a cabo en municipios relativamente populosos donde hay centros de educación secundaria. Aun así, la mayor parte de las localidades seleccionadas concentran a estudiantes de los pueblos más pequeños de la misma comarca. Igualmente, las siete ciudades seleccionadas en el ALeCMan para ampliar el espectro diastrático están representadas ampliamente en el léxico disponible.

El último aspecto que hay que destacar es la adaptación de los visores de resultados. Los materiales del ALeCMan se plasman en mapas donde aparecen las variables léxicas y su trascripción fonética, los del léxico disponible en relaciones de palabras, agrupadas por categorías semánticas y ordenadas por índice de disponibilidad. Se ha hecho necesaria una reformulación de los datos del léxico disponible en torno a la procedencia de los informantes, de forma que también sea posible observar de modo bidimensional la extensión de los términos en el espacio geográfico.

\subsection{Observaciones generales}

Desde un punto de vista global, el primer paso es contrastar las zonas donde se han documentado los términos comunes a ambos inventarios léxicos. La mayor parte de las unidades que permiten el cotejo pertenece a categorías semánticas relacionadas con el mundo rural: animales, plantas y trabajos del campo. No es intención de estas páginas mostrar de forma exhaustiva todas las coinci-

\footnotetext{
${ }^{22}$ Las localidades del ALeCMan pueden consultarse en la página electrónica (vid. nota 13) y las del léxico disponible de Castilla-La Mancha en el mapa 2. Las municipios no coincidentes son: en Toledo, Los Navalmorales y Torrijos; en Albacete, Villarrobledo; en Cuenca, Landete; en Guadalajara, Cifuentes; y en Ciudad Real, Almadén, Porzuna, Viso del Marqués, Almodóvar del Campo, Alcázar de San Juan, Valdepeñas y Manzanares.
} 
dencias léxicas de ambas investigaciones, sino presentar una selección de ejemplos representativos que ilustren las posibilidades del enfoque planteado.

La comparación sistematizada ha sido llevada a cabo de dos formas: 1) observando la proyección de términos aislados y 2) analizando la distribución de conjuntos de términos sinonímicos que aluden al mismo concepto y que tienen una marcada distribución geográfica. Veamos algunos ejemplos de diferente consideración relativos al primer modo de análisis:

a) El término espliego para la lavanda (Espica angustifolia) según el ALeCMan (mapa 142, $\mathrm{II}^{23}$ ) se documenta de forma exclusiva en la mitad oriental de la comunidad (provincias de Guadalajara, Cuenca y Albacete y zonas fronterizas de Toledo y Ciudad Real). De forma similar, en el léxico disponible nueve de las diez apariciones de espliego se producen en esta área, especialmente en Guadalajara. En el resto de la comunidad solo aparece un testimonio esporádico en Puertollano.

b) El caso de avión (Delichon urbica) y de vencejo (Apus apus) también ofrece similitudes entre ambas investigaciones. En esta ocasión son dos los mapas del ALeCMan que han de ser comparados simultáneamente, ya que avión aparece en diferentes muestras cartográficas (mapas 28 y 30, I), donde alterna con vencejo. Los lugares donde se ha documentado avión en el léxico disponible (sierra de Alcaraz y en centro-oeste de Ciudad Real) son también aquellos donde el ALeCMan marca la variación. Vencejo es un término producido en el léxico disponible casi en exclusiva por ocho participantes de la provincia de Cuenca y un informante de Torrijos (norte de Toledo). En el ALeCMan la extensión de esta voz es más amplia —a excepción de Albacete, está presente en toda la comunidad.

c) Según el mapa 22, I del ALeCMan, gazapo 'cría de la liebre' se extiende por una franja que discurre desde el centro-norte de Toledo a través de La Mancha central hasta el oeste de Albacete. Es ahí (Alcaraz) y en el área manchega (Tarancón) donde es incluida en las listas del léxico disponible.

d) Solo hay un caso de aparición del vocablo mojón en el léxico disponible de Castilla-La Mancha, en Cifuentes (Guadalajara) y éste coincide de nuevo con el área de extensión de este término en el atlas lingüístico (provincias de Cuenca y Guadalajara).

e) La forma ababol (amapola en el resto de la región) ha sido producida por un estudiante de Alcaraz (Albacete). Esta documentación se sitúa también dentro de la zona de extensión del término en el ALeCMan (mapa 134, II, provincias de Guadalajara, Cuenca y Albacete).

${ }^{23}$ Se hará referencia al número de mapa (números arábigos) y al cuestionario (números romanos) tal y como aparece en la página electrónica. 
f) En algunos ejemplos los datos del léxico disponible complementan, no solo confirman, los datos del ALeCMan. El término abejaruco para referirse al Bombus o abejorro se documenta en el ALeCMan en el suroeste de Ciudad Real (Valdeverdeja y Servilleja de la Jara) y en el oeste de Guadalajara (Cogollado, Peñalver, Loranca de Tajuña y Pastrana). Sin embargo, en el léxico disponible no es actualizado por hablantes de esas zonas, sino de las colindantes. Por tanto, abejaruco amplía su extensión a la franja central del territorio, uniendo las dos islas léxicas reflejadas en el ALeCMan y creando un continuum en el uso del término.

En algunos casos el léxico disponible pone de manifiesto también las variaciones fonéticas y/o morfológicas que se ven reflejadas en la cartografía del atlas lingüístico.

g) Así, en el mapa 42, II, de paloma torcaz (Columba palumbus) alternan las formas torcal, torcaz y torcazo. El léxico disponible documenta la segunda y la tercera, en las zonas donde el ALeCMan incluye estas variantes concretas: torcaz en Puertollano (Ciudad Real), Almansa (Albacete) y Los Navalmorales (Toledo) y torcazo en el suroeste de Albacete.

h) Los casos de aféresis de (a)bubilla que se registraron en el léxico disponible (Almansa en Albacete y Cifuentes en Guadalajara) corresponden a zonas donde se cartografía este fenómeno en el ALeCMan (mapa 58, I).

i) Otro de los grupos de términos cuya observación completa el panorama presentado es: azada, azadón, azadilla y escavillo. Estas cuatro formas, que aluden a diferentes herramientas para la labor agrícola, se intercalan en tres mapas del ALeCMan, el 120 (azada), 121 (azadón) y 128 (escardillo) del cuestionario I. En los resultados del ALeCMan se puede observar cómo en los tres mapas, que en principio se refieren a utensilios diferentes, también fluctúan estas formas. Azada se extiende de forma homogénea por toda la comunidad, así también lo reflejan los informantes del léxico disponible, si bien hay zonas donde su uso es casi exclusivo (Albacete), mientras que en otras se combina con la presencia de azadón y azadilla. Es destacable que los registros escritos de léxico disponible también manifiestan las variaciones gráfico-fonéticas que presentan los mapas. Dos informantes de La Mancha central y uno del sur de Albacete escriben azá: la pérdida de la $-d-$ intervocálica en el ALeCMan es casi sistemática en esas zonas. La extensión de este fenómeno es todavía más elevada en el caso de azadilla y azadón. Dos de los cuatro informantes que escriben azadilla marcan la pérdida de la dental, en un caso omitiendo la letra, azaílla, y en otro sustituyendo la grafía $-d-$ por una $-h-$, azahilla, quizá marcando que el hablante es consciente de la existencia (u omisión) de un sonido en la lengua oral. Las documentaciones escritas de esta palabra se producen en localidades donde también se da la datación cartográfica (centro de Ciudad Real y norte de La Mancha). En tercer lugar, los resultados del léxico disponible 
nos indican que azadón es un término más limitado geográficamente. No hay ningún registro en Albacete, ni en el oeste de Cuenca y tan solo dos en Guadalajara. Tal y como refleja el ALeCMan, azadón es propio de la zona central y oriental de la comunidad, especialmente de la comarca manchega y de Toledo. Aunque en los listados editados del léxico disponible aparece únicamente la forma azadón, los informantes utilizaron en las encuestas una amplia variedad de grafías que aportan información sobre su pronunciación (y que también incluyen errores ortográficos como la $h$-inicial) ${ }^{24}$. De las 33 documentaciones de azadón, 12 marcan la pérdida de la $-d$ - intervocálica y escriben azaón (10) o azahón (2). Un registro tan elevado de este fenómeno $(36,6 \%$ ) en una encuesta escrita, realizada a hablantes jóvenes en un contexto académico y que favorece el léxico estándar y formal, indica la vitalidad tan elevada de esta pérdida fonética en este grupo de términos. Relacionada semánticamente con estas voces aparece en las listas la variante dialectal escavillo. El mapa del ALeCMan para escardillo sitúa la forma diferencial en Albacete (también el Diccionario de la Real Academia Española ${ }^{25}$ la reseña con la marca dialectal Alb.). Los estudiantes que escriben esta palabra en el léxico disponible son de Hellín (Albacete) y de Tarancón (Cuenca). Queda así ampliada su extensión con respecto al ALeCMan.

Hay que tener en cuenta que el hecho de que los informantes que participaron en la encuesta de léxico disponible no produzcan una palabra no significa que no la conozcan o no la usen, tan solo que no se encuentra entre las más disponibles de su comunidad. El ALeCMan, sin embargo, pregunta directamente por conceptos que llevan a los términos léxicos buscados. Por consiguiente, no es tan relevante la falta de un término en las listas de disponibilidad, como la documentación en lugares más allá de los límites que presenta el ALeCMan, ya que es síntoma de la progresiva extensión de las voces (o de que, por causas desconocidas, no fueron consignadas).

La correspondencia encontrada entre los resultados del léxico disponible y los mapas del ALeCMan puede estudiarse de forma más detallada en el segundo caso que se planteaba al comienzo de §3.2: la distribución de los conjuntos de palabras sinónimas o cuasisinónimas que designan la misma realidad. En los siguientes apartados se estudian detalladamente dos de estos ejemplos: las formas cerdo, gorrino, cochino y guarro para Sus scrofa domestica y las palabras para designar la parte baja del abdomen, vientre, tripa y barriga.

${ }^{24}$ En toda la investigación se observan inseguridades gráficas de este tipo, especialmente en aquellas palabras que no se encuentran habitualmente en los registros escritos.

25 Real Academia Española, Diccionario de la Lengua española, www.rae.es, 2001. A partir de ahora DRAE. 


\subsection{Cerdo, gorrino, cochino y guarro}

En la tabla 1 aparecen los cuatro términos ordenados según los índices de disponibilidad obtenidos en el centro de interés Animales (el rango hace referencia a su posición en la lista). Las dos formas más frecuentes, cerdo y gorrino, fueron recogidas tanto en singular como en plural.

TABLA 1

\begin{tabular}{|l|c|c|}
\hline \multicolumn{1}{|c|}{ Vocablo } & Rango & Disponibilidad \\
\hline cerdo/s & 15 & 0,2269 \\
\hline gorrino/s & 106 & 0,0268 \\
\hline guarro & 149 & 0,0123 \\
\hline cochino & 162 & 0,0104 \\
\hline
\end{tabular}

Cerdo es la palabra más disponible y también la que posee una distribución más homogénea en el territorio castellano-manchego. Las otras tres variantes, sin embargo, solo han sido documentadas en unas zonas muy concretas que coinciden plenamente con las reflejadas en ALeCMan (mapa 1, I). En el mapa 1 ilustramos la distribución geográfica de estos tres términos. Solo hemos tenido en cuenta las menciones en el centro de interés Animales, aunque también aparece en El campo (gorrino y guarro) y en Alimentos y Bebidas (cochino).

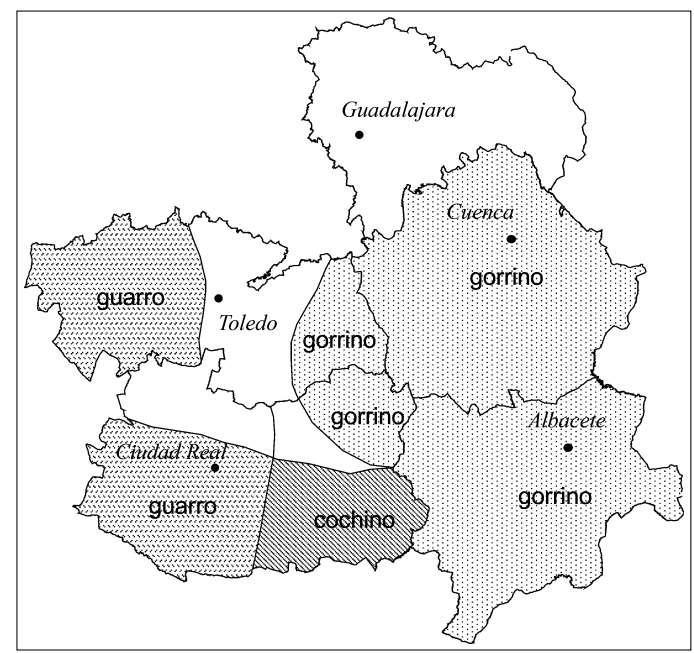

MAPA 1: Extensión de los términos guarro, cochino, gorrino en el léxico disponible de Castilla-La Mancha (Animales) 
a) Cochino ha sido producida por trece estudiantes del centro-sureste de la provincia de Ciudad Real (Alcázar de San Juan, Viso del Marqués, Valdepeñas y Ciudad Real26); en el ALeCMan se registra en el sur de la provincia ciudadrealeña.

b) Guarro ha sido producida por cuatro informantes de la mitad oeste de la provincia de Toledo (Navalmorales, Talavera y Torrijos) y por once informantes del oeste y del centro de Ciudad Real (Puertollano, Almadén, Porzuna, Ciudad Real y Almodóvar del Campo). Estos resultados también coinciden con el mapa del ALeCMan, si bien guarro se extiende en ambas provincias hacia zonas más occidentales.

c) La forma gorrino ha sido documentada en la provincia de Albacete en ocho ocasiones, en Cuenca en trece ocasiones y nueve veces más en los pueblos más orientales de las provincias de Toledo (Quintanar de la Orden y Villacañas) y nororientales de Ciudad Real (exclusivamente en el municipio de Alcázar de San Juan). De nuevo la muestra coincide con el ALeCMan - en este último caso gorrino aparece por toda la franja oeste de Ciudad Real, no solo por La Mancha.

En el mapa 1 también se puede observar que hay dos zonas donde no se registró ninguna de las voces diferenciales (guarro, cochino y gorrino) sino solo la forma que podríamos considerar más neutra, cerdo. Estas son el centro de Toledo y Guadalajara, ambas áreas de influencia madrileña. Será precisamente Guadalajara el territorio con un menor promedio de dialectalismos por informante en el léxico disponible.

A modo de resumen, en este conjunto de términos, cerdo sería la variante estándar de la comunidad —entendida como unidad léxica sin marca local, compartida por todos los hablantes - mientras que gorrino, guarro y cochino, formarían la matriz de uso dialectal superpuesta (utilizo aquí la terminología propuesta por Carriscondo ${ }^{27}$ para el estudio lexicográfico de las variedades noestándar). Estas tres formas son consideradas en este estudio particular como localismos, ya que su uso se circunscribe a una parcela muy concreta del territorio $^{28}$. Esto no quiere decir que en otras zonas peninsulares alguna de ellas

${ }^{26}$ Los centros de la capital recogen informantes de toda la provincia y por lo tanto, es justificable que entre los informantes de Ciudad Real capital se encuentre tanto la forma cochino (más propia del sur de la provincia) como guarro (de la zona más occidental).

${ }^{27}$ Francisco Manuel Carriscondo Esquivel, La lexicografía en las variedades no-estándar, Jaén, Publicaciones de la Universidad de Jaén, 2001.

${ }^{28}$ Otro de los conjuntos de sinónimos relacionados con este grupo, cuya extensión geográfica se manifiesta paralela en las dos investigaciones, es lechón-cochinillo-guarrillo. El primer término se centraliza en La Mancha, el segundo se ha recogido de forma esporádica en el norte de Toledo (territorio fronterizo con el sur de Castilla y León donde esta forma es de uso extendido) y guarrillo en el este de Ciudad Real, área de extensión de guarro. 
pueda funcionar como término estándar. De hecho, el $D R A E$ remite en los tres casos a la entrada cerdo sin añadir ninguna marca de uso restringido.

\subsection{Vientre, tripa y barriga}

Los términos vientre, tripa y barriga obtienen en las listas del vocabulario disponible castellano-manchego del centro de interés Partes del Cuerpo los índices reflejados en la tabla 2 .

TABLA 2

\begin{tabular}{|l|c|c|}
\hline \multicolumn{1}{|c|}{ Vocablo } & Rango & Disponibilidad \\
\hline barriga & 67 & 0,0275 \\
\hline tripa $^{29}$ & 80 & 0,0205 \\
\hline vientre & 111 & 0,0108 \\
\hline
\end{tabular}

Este grupo de palabras presenta un esquema relacional diferente a cerdo (§3.3.) ya que no hay un término neutro común a todo el territorio y una matriz de variantes geográficas, sino que las tres formas aparecen en toda la región articuladas en forma de predominios léxicos. Esto no es óbice para observar las tendencias geográficas que se muestran en los resultados y compararlas con los datos del atlas linguiístico.

$\mathrm{Si}$ describimos detalladamente el alcance territorial de cada término en el léxico disponible, vientre se documenta en las cinco provincias, pero es muy escasa en Cuenca ( 1 informante). Tripa también se documenta en las cinco zonas pero disminuye su presencia en Albacete ( 1 informante) y en Guadalajara ( 2 informantes). Barriga es el término con una distribución territorial más definida pues solo aparece en Guadalajara, Ciudad Real y Albacete, mientras que no documenta ningún registro en Cuenca ni en Toledo - y aun así consigue ser la forma más disponible de la región.

Los resultados del ALeCMan (mapa 314, II) también muestran un panorama más complejo que en el caso de cerdo. A continuación comparo la distribución por provincias.

En Toledo y en Cuenca predomina la forma tripa, pero también se documenta abundantemente vientre, especialmente en la zona suroeste de la provin-

29 Solo se han contabilizado las veces que aparece tripa en singular, ya que se considera que la forma tripas alude a una parte del cuerpo diferente. 
cia conquense, en la ribera del Tajo y en el centro de Toledo. En estas dos provincias la aparición de barriga es muy esporádica —en el diccionario de léxico disponible, inexistente. El informante que en Cuenca anota vientre, es precisamente de Tarancón, zona que en el mapa del ALeCMan también refleja este término. Los informantes de Toledo que escriben vientre, son de Ocaña y Talavera de la Reina, municipios próximos a la ribera del río.

En Albacete predomina barriga, al igual que en el vocabulario disponible, pero también se documenta tripa en zonas periféricas como la sierra de Alcaraz y la ribera del Júcar, esta última cercana a la influencia de la elección léxica conquense. La aparición de vientre es infrecuente. El único informante que escribe tripa en el léxico disponible es de Albacete capital. También ahí aparece la forma vientre en dos ocasiones, por ser lugar donde confluyen las hablas comarcales. El tercer estudiante que escribe vientre es de Hellín, municipio que en el ALeCMan también recoge esta forma.

En Ciudad Real los términos barriga y vientre se reparten por toda la provincia según los datos del ALeCMan. Mucho menos frecuente es la respuesta tripa: tampoco abunda en el léxico disponible (solo en la comarca de Porzuna y Ciudad Real capital).

Hasta aquí los resultados de ambos trabajos muestran continuas similitudes. Sin embargo, en Guadalajaran según el ALeCMan predomina el término tripa, seguido de barriga y en menor medida, vientre, mientras que los datos del diccionario de léxico disponible aportan cuatro menciones de barriga, dos de vientre y dos de tripa.

Considerando tanto estos resultados como los de la matriz dialectal de cerdo, la provincia de Guadalajara es el territorio donde los datos del ALeCMan y del léxico disponible son más distantes ${ }^{30}$.

\subsection{Discusión}

Desde un punto de vista general, se puede concluir que los diccionarios de léxico disponible de áreas extensas permiten elaborar mapas lingüísticos que reflejan las preferencias léxicas diatópicas y que estos materiales coinciden, en cierta medida, con los recogidos en las obras geolingüísticas de referencia, como es el ALeCMan en el caso de Castilla-La Mancha. Los ejemplos aportados demuestran, tal y como propusieron Borrego Nieto y Fernández Juncal ${ }^{31}$,

30 Esto puede deberse a las características idiosincrásicas de los centros de educación secundaria que participaron en el proyecto, a pesar de que su selección se hizo de forma razonada: los 80 informantes de Guadalajara se distribuyen equilibradamente en ámbito urbano (40, Guadalajara) y ámbito rural (40, Cifuentes y Sigüenza).

31 Julio Borrego Nieto y Carmen Fernández Juncal, «Léxico...», op. cit. 
que la herramienta metodológica de la disponibilidad léxica sirve para reflejar fiablemente las preferencias dialectales de una región. Aun así, el estudio piloto que aquí se presenta no deja de ser limitado en cuanto al número de unidades léxicas estudiadas y al espacio geográfico, por lo que convendría comprobar estos resultados en otros ámbitos temáticos y otras áreas territoriales.

Aun así, hay que tener en cuenta que los perfiles sociales de los sujetos de ambas investigaciones son distintos: mientras que en el ALeCMan predominan los adultos de edad avanzada sin instrucción, los resultados del léxico disponible son de jóvenes preuniversitarios. Esta diferencia metodológica puede ser tomada no como una desventaja, sino como un índice de la vitalidad de los términos, ya que si ambos repertorios coinciden, se comprueba que las unidades léxicas poseen proyección futura: además de formar parte del acervo léxico de la población adulta, son parte del léxico disponible de los hablantes jóvenes de las mismas zonas. Esta significación temporal se acentúa al considerar que las recogidas de los materiales se hicieron con quince años de diferencia. Así, se observa cómo las generaciones adultas (abuelos y padres) transfieren el sistema léxico de una comunidad a las nuevas generaciones (los hijos) y cómo esto conlleva una estabilidad en el trazo de las isoglosas léxicas en los términos más disponibles del léxico cotidiano.

\section{DiSTRIBUCIÓN GEOGRÁFICA DE LOS DIALECTALISMOS DEL LÉXICO DISPONIBLE}

\subsection{Cuantificación en las zonas dialectales}

Dos estudios previos abordan la presencia de términos dialectales en el léxico disponible de Castilla-La Mancha: el primero analiza los regionalismos en el diccionario de léxico disponible desde un punto de vista general, con el objetivo de documentar todas las palabras aparecidas en los listados y de establecer la proporción total de regionalismos ${ }^{32}$; el segundo presenta desde un punto de vista sociolingüístico la actualización de dicho léxico dialectal en diferentes grupos sociales $^{33}$. En este segundo trabajo se destaca cómo, en algunas ocasiones, la variable geográfica interfiere con variables sociales tales como el tipo de centro educativo (público o privado) o la consideración de las comunidades rurales o urbanas. Se manifiesta así el hecho de que en el estudio de la variación léxica no es fácil deslindar lo geográfico, de lo social o lo situacional ${ }^{34}$.

\footnotetext{
32 Natividad Hernández Muñoz, Hacia..., op. cit., págs. 365-402.

${ }^{33}$ Natividad Hernández Muñoz, «Aspectos sociolectales del léxico dialectal», Spanish in Context, 2009, 6, 2, págs. 224-248.

${ }^{34}$ Francisco Moreno Fernández, «Los estudios...», op. cit.
} 
Para continuar con el interés despertado por estos resultados, surgió la posibilidad de analizar la carga dialectal que poseían las diferentes zonas lingüísticas identificadas dentro de Castilla-La Mancha. Al realizar este proceso, indirectamente se comprobaba si las divisiones en las áreas dialectales tradicionales funcionaban como variables de clasificación geográfica para predecir una aparición más o menos abundante de léxico regional en las palabras disponibles. De esta forma se completaban las posibilidades de análisis dialectal que ofrecía la herramienta metodológica de la disponibilidad léxica.

Como se destacaba en $§ 2$, los estudios clásicos sobre las hablas dialectales de Castilla-La Mancha trazan isoglosas que no concuerdan con los límites provinciales, sino que están ligados a las comarcas geográfico-culturales. El mapa 2 muestra las zonas dialectales consideradas para el análisis. Están basadas en la división que propone Moreno Fernández para Castilla La Nueva ${ }^{35}$ con algunos cambios estructurales. Debido a que para el léxico disponible de CastillaLa Mancha no se realizaron encuestas en la zona madrileña, se han unificado la zona noroeste y la zona de Madrid de Moreno Fernández en una sola, llamada noroeste a partir de ahora; y, en contraposición, la zona norte pasa a llamarse noreste, de forma que el número total de áreas dialectales es de seis: (1) La Mancha, (2) Oeste, (3) Sureste, (4) Este, (5) Noroeste y (6) Noreste.

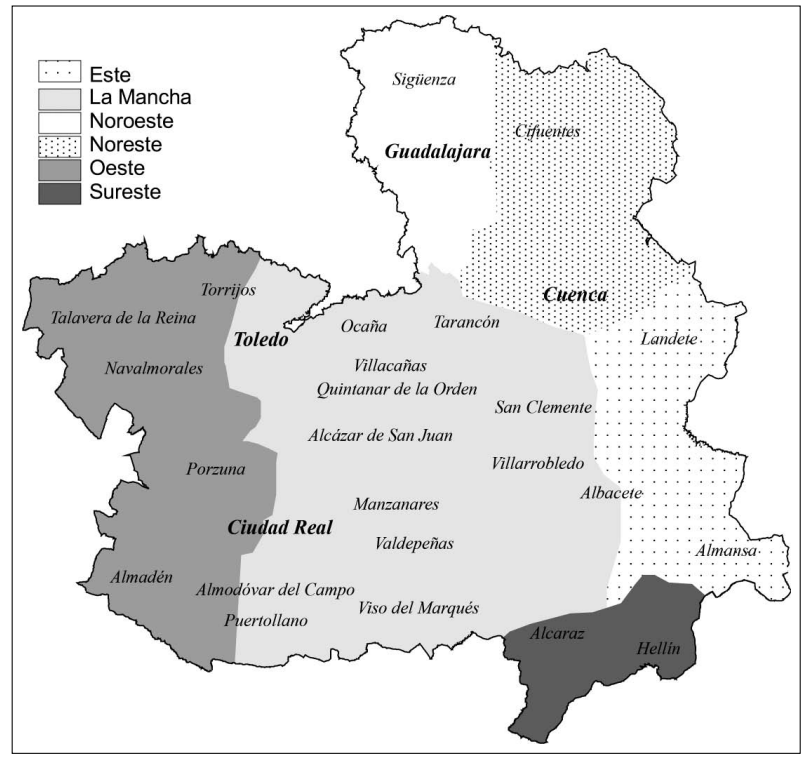

MAPA 2. Zonas dialectales de Castilla-La Mancha.

\footnotetext{
35 Francisco Moreno Fernández, «Castilla...», op. cit., pág. 230.
} 
Para valorar la presencia de los dialectalismos en las diferentes áreas lingüísticas se van a utilizar los conceptos de amplitud y vitalidad propuestos por Borrego Nieto ${ }^{36}$. La amplitud de un fenómeno lingüístico es la extensión dentro de su campo de acción específico, esto es, el número de manifestaciones de dicho fenómeno en el conjunto observado. Para evaluar la amplitud de los dialectalismos se ha considerado el porcentaje de términos dialectales en el conjunto de vocablos que forman en léxico disponible (Tabla 3). La vitalidad hace referencia al grado mayor o menor de uso de un elemento dentro de la comunidad. Así, la vitalidad de los dialectalismos vendrá marcada por el número de veces que cada informante produzca una de estas unidades léxicas (Tabla 4). La posibilidad de cuantificar ambos aspectos ofrece la ventaja de observar el fenómeno

TABLA 3

Amplitud de los regionalismos

\begin{tabular}{|l|c|c|c|}
\hline & Vocablo & Rango & Disponibilidad \\
\hline 1. La Mancha & 57 & 6459 & $0,88 \%$ \\
\hline 2. Oeste & 59 & 7398 & $0,80 \%$ \\
\hline 3. Sureste & 30 & 2927 & $1,02 \%$ \\
\hline 4. Este & 32 & 4351 & $0,73 \%$ \\
\hline 5. Noroeste & 13 & 4208 & $0,31 \%$ \\
\hline 6. Noreste & 15 & 3265 & $0,46 \%$ \\
\hline
\end{tabular}

TABLA 4

Vitalidad de los regionalismos

\begin{tabular}{|l|c|c|c|}
\hline & Menciones & Informantes & Promedio \\
\hline 1. La Mancha & 106 & 200 & 0,53 \\
\hline 2. Oeste & 97 & 260 & 0,37 \\
\hline 3. Sureste & 52 & 40 & 1,30 \\
\hline 4. Este & 48 & 80 & 0,60 \\
\hline 5. Noroeste & 18 & 60 & 0,30 \\
\hline 6. Noreste & 16 & 40 & 0,40 \\
\hline
\end{tabular}

36 Julio Borrego Nieto, Norma y dialecto en el sayagués actual, Salamanca, Ediciones Universidad de Salamanca, 1983. 
dialectal desde una perspectiva más completa. Tal y como manifiesta Borrego Nieto: «la amplitud de los fenómenos linguísticos suele quedar bien reflejada en los estudios dialectales tradicionales, pero no siempre sucede lo mismo con la vitalidad» ${ }^{37}$, y esta última es más significativa en cuanto a la perspectiva diastrática adoptada en los estudios de dialectología social.

La zona sureste de la comunidad es la que presenta un mayor grado tanto de vitalidad como de amplitud de uso de términos de carácter regional. Si el porcentaje de palabras dialectales $(1,02 \%)$ lo sitúa por encima de las restantes áreas, el promedio de menciones es todavía más elevado $(1,30)$, lo que indica que cada informante produjo, al menos, un término dialectal. Esta zona incluye las poblaciones del sur de Albacete: Alcaraz en la sierra y Hellín en el corredor murciano.

La zona con una menor presencia de carga dialectal, tanto en el caso de la vitalidad de los términos $(0,30)$ como en la amplitud $(0,31 \%)$, es el área noroeste, que comprenden las localidades de Guadalajara capital y Sigüenza.

El resto de parcelas territoriales posee unos índices equilibrados entre sí. Entre ellos, destaca la amplitud de los regionalismos en La Mancha y en la zona oeste (será también La Mancha la que presente un mayor número de términos locales exclusivos §4.2.1.). Es en estas zonas donde se pone de manifiesto la diferencia entre los dos índices utilizados para valorar la presencia dialectal. Mientras que son comarcas donde la extensión del léxico dialectal es elevada, la vitalidad, es decir, el uso (producción) dentro de la comunidad de hablantes jóvenes, es limitada.

Hay que puntualizar que estos promedios no representan un índice para valorar el léxico dialectal de estas zonas desde un punto de vista general, sino que son una manifestación parcial de la realidad dialectal de la comunidad. Indican la extensión y la vitalidad de los regionalismos en el conjunto de las palabras más disponibles de la población joven (aquellas palabras que más fácilmente son producidas en torno a diferentes temas). Cobran valor, precisamente,

TABLA 5

Estadísticos del ANOVA

\begin{tabular}{|l|c|c|c|c|c|}
\hline & $\begin{array}{c}\text { Suma de los } \\
\text { cuadrados }\end{array}$ & $\begin{array}{c}\text { Grados de } \\
\text { libertad }\end{array}$ & $\begin{array}{c}\text { Media al } \\
\text { cuadrado }\end{array}$ & $F$ & Probabilidad \\
\hline Intergrupos & 35,638 & 5 & 7,128 & 12,182 &, 000 \\
\hline Intragrupos & 394,349 & 674 &, 585 & & \\
\hline Total & 429,987 & 679 & & & \\
\hline
\end{tabular}

37 Ibíd., pág. 13. 
al considerar que estos recuentos no favorecen el léxico de carácter regional, sino el léxico estándar con cierto grado de formalidad.

Para completar la sección dedicada a los índices cuantificadores, se ha realizado un análisis de la varianza (ANOVA) donde se utiliza como variable dependiente el número de dialectalismos dados por cada informante y como variable independiente el área geográfica de procedencia. Tal y como aparece en la tabla 5, el resultado muestra cómo la diferencia entre la producción cuantitativa de las áreas dialectales es también significativa desde el punto de vista estadístico $(\mathrm{p}<0.05)$, por lo que la división comarcal propuesta podría predecir qué vocabularios disponibles consignarían un mayor número de voces regionales.

$\mathrm{Al}$ observar estos resultados surge la siguiente pregunta: ¿por qué razón la zona sureste de Castilla-La Mancha posee una cantidad tan alta de léxico dialectal?, ¿existe alguna característica ajena a las propias peculiaridades léxicas? En primer lugar, es la única división territorial donde todos los informantes son de núcleos rurales, no urbanos. En un trabajo anterior se observaba que la variable urbano-rural era un fuerte condicionante de la presencia dialectal ${ }^{38}$, por lo que se podría llegar a pensar que esta característica condiciona los resultados. Para comprobar si efectivamente sucedía esto, se realizó un nuevo análisis estadístico suprimiendo a los informantes de procedencia urbana que podrían reducir los promedios. Los resultados estadísticos son similares, la variable comarca geográfica continúa prediciendo significativamente la aparición de voces dialectales $(\mathrm{p}<0.05)$ y la zona sureste es la que mayor promedio de dialectalismos obtiene $^{39}$. La misma prueba se efectuó eliminando a los informantes de colegios privados (ya que la variable público-privado también afectaba significativamente al promedio de dialectalismos) y el resultado fue similar. La parcelación en zonas dialectales continúa siendo significativa desde el punto de vista estadístico $(\mathrm{p}<0.05)$, con un promedio más alto en la zona sureste ${ }^{40}$.

\subsection{Apuntes cualitativos}

A continuación se presenta una selección de los términos dialectales exclusivos de cada una de las seis áreas léxicas propuestas. Esta relación no incluye los regionalismos extendidos por todo el territorio castellano-manche-

\footnotetext{
38 Natividad Hernández Muñoz, «Aspectos...», op. cit.

39 Promedios sin los informantes de colegios de capitales de provincia: La Mancha $(0,52)$, Oeste $(0,39)$, Sureste $(1,33)$, Este $(0,80)$, Noroeste $(0,25)$ y Noreste $(0,45)$. En este caso el único índice que aumenta considerablemente es del de la zona este, que en los promedios generales había obtenido 0,60. En el resto la variación es mínima.

40 Promedios sin los informantes de colegios privados: La Mancha $(0,52)$, Oeste $(0,40)$, Sureste $(1,33)$, Este $(0,60)$, Noroeste $(0,15)$, Noreste $(0,45)$. En este caso la variación más relevante es la reducción de los dialectalismos en la zona noroeste (promedio general 0,30 ).
} 
$\mathrm{go}^{41}$, solo aquellos dados por dos o más informantes en una sola de las parcelas territoriales. Según la valoración cualitativa, que tiene íntima relación con el concepto de extensión considerado en el apartado anterior, La Mancha emerge como el área más dialectal.

\subsubsection{La Mancha}

ablendar (ablentar, DUE ${ }^{42}$ 'aventar'),

amorterar $\left(\right.$ Calero $^{43}$ 'poner tierra todo alrededor de la cepa para evitar que se hiele en invierno'),

cubertera ('recipiente para cubiertos'), espumadera ('utensilio de cocina para retirar la espuma'),

follonar (esfollonar, Sánchez Miguel ${ }^{44}$ 'quitar las hojas más pequeñas de la parra'),

glorio (gloria, DRAE 'estrado hecho sobre un hueco abovedado en cuyo interior se quema paja u otro combustible para calentar la habitación; hornillo dispuesto a calentarse y cocer las ollas'),

horquillo ( $D E A$ 'horca agrícola de madera con dos púas') y semillar (DRAE 'sembrar, esparcir semillas').

\subsubsection{Oeste}

carpesano ('carpeta grande con anillas gruesas para archivar'), gradear (DEA 'gradar, pasar la grada por un campo') y pimientera ('recipiente para la pimienta').

\footnotetext{
41 Para consultar la relación completa de voces regionales y su distribución social véase Natividad Hernández Muñoz, Hacia..., op. cit. y «Aspectos...», op. cit. Hay que señalar que entre estos términos hay tanto variantes léxicas como variantes morfológicas: por ejemplo el caso de aceitero marcado como regional en el Diccionario de Español Actual (Manuel Seco, Olimpia Andrés y Gabino Ramos, Madrid, Aguilar, 1999; DEA a partir de ahora) o verbos en los que se alternan los prefijos es-, des- considerados por Moreno Fernández («Castilla...», op. cit., pág. 223) como peculiaridad de los sociolectos castellano-manchegos más bajos). También se incluyen palabras que no han sido documentadas en los diccionarios generales ni en los diferenciales, pero que son de uso común en la región. En las definiciones se incluye la referencia a las obras lexicográficas de donde han sido tomadas y los términos de referencia en el caso de las variantes morfológicas. Si no aparece ninguna indicación, la definición es mía.

${ }^{42}$ María Moliner, Diccionario de Uso del Español, segunda edición, Madrid, Gredos, 1998.

43 José Luis Calero López de Ayala, Vocabulario dialectal de La Mancha conquense, Cuenca, Sección de publicaciones de la Diputación provincial de Cuenca, 1995.

44 J. M. Sánchez Miguel, Diccionario del habla toledana, Toledo, Instituto Provincial de Investigaciones y estudios Toledanos, Diputación provincial, 1998.
} 


\subsubsection{Sureste}

ajo de harina (Serna ${ }^{45}$ 'guiso que se hace sofriendo patatas, ajos, tomate, harina y pimentón, añadiendo también el agua para la cocción de todo ello'), ajo de matazón (Chacón ${ }^{46}$ 'guiso hecho con hígado de cerdo y almendras de piñón, todo ello espolvoreado con canela'),

ajopringue $\left(\right.$ Calero $^{47}$ 'plato que preparan con la pringue o manteca de conservar chorizos además de patatas, carne y agua en la que deslíen varios ajos en el mortero'),

espollizar ('quitar pollizos, brotes que salen al pie del árbol, especialmente del olivo'),

güeña (DRAE 'embutido compuesto de las vísceras del cerdo, excepto el hígado y algunas carnes gordas de desperdicio de los demás embutidos, picado todo y adobado con ajos, pimentón, pimienta, clavo, sal, orégano y otras especias') y

migas ruleras (Calero ${ }^{48}$ 'versión mas humilde [de las migas] ya que se limitan a freír el pan duro, troceado en dados, previamente humedecido sin añadir ningún ingrediente salvo la sal hasta que quedan muy sueltas').

\subsubsection{Este}

bajocas (DUE 'en muchas regiones de España, judía verde'), cubiertera ('recipiente para cubiertos'),

espuntar (despuntar, 'cortar una parte del sarmiento para que no lo rompa el viento') $\mathrm{y}$,

peúcos (Yunta ${ }^{49}$ 'calcetines de lana para llevar debajo de las albarcas', Sánchez Miguel $^{50}$ 'calcetines abotonados para el campo').

\subsubsection{Noroeste \\ cipotero (DRAE 'ribazo, mojonera') y sobreplato ('por oposición a bajoplato, plato de arriba').}

45 José Salustiano Serna, Cómo se habla en La Mancha. Diccionario manchego, Villarrobledo, Imprenta Cervantes, 1981.

46 Teudiselo Chacón, El habla de la Roda (contribución al estudio del habla manchega), Albacete, Instituto de Estudios Albacetenses de la Excma. Diputación de Albacete, 1981.

47 José Luis Calero López de Ayala, Vocabulario..., op. cit.

48 Ibíd.

49 Pedro Yunta Martínez, Conquensismos, Cuenca, Caja de Ahorros de Cuenca, 1978.

50 J. M. Sánchez Miguel, Diccionario..., op, cit. 


\subsubsection{Noreste}

chanela (DUE 'zapatillas sin talón') y

pota (DEA 'olla más baja y ancha que el pote').

Asimismo, hay que destacar que se observa una gran conexión en cuanto al léxico dialectal de La Mancha y de la zona oeste, ya que existe un grupo de términos que comparten estos dos territorios y que no aparecen en ninguna otra de las áreas dialectales establecidas: chuponar ('quitar chupones', chupón $D R A E$ 'vástago que brota de las ramas principales que chupa la savia y amarga el fruto'; 13 menciones en La Mancha, 7 en el oeste), aceitero (DEA 'aceitera'; 7 menciones en La Mancha, dos en el oeste), vinagrero (vinagrera; 5 menciones en La Mancha, tres en el oeste), ablentar (DUE 'aventar'; 1 mención en La Mancha —donde aparece también la forma ablendar - y dos en el oeste) y espumillera ('utensilio de cocina para retirar la espuma'; dos menciones en La Mancha, tres menciones en el oeste).

Otros términos que habría que destacar son aquellos que se reducen a una de las provincias administrativas: en Cuenca rayolar ('quitar los sarmientos tiernos que no tienen flor de uva') y resolí (DRAE 'aguardiente con canela, azúcar y otros ingredientes olorosos'), en Guadalajara chanela (vid. §4.2.6.), en Albacete zompo (Calero ${ }^{51}$ 'trompo') y leja (DRAE 'vasar'), en Ciudad Real carpesano (vid. \$4.2.2.) y en Toledo horquillo (vid. \$4.2.1.) y espumadera ('utensilio de cocina para retirar la espuma').

\section{CONCLUSIONES}

De acuerdo con los objetivos de este trabajo, se ha mostrado cómo la metodología de obtención del léxico disponible se puede aplicar al estudio concreto de las áreas dialectales de una determinada zona tal y como proponían Borrego Nieto y Fernández Juncal. En este caso, la región castellano-manchega ha servido como modelo de análisis a través de la comparación de su vocabulario disponible con otras obras clásicas de la dialectología y la geolingüística españolas.

Además de esta conclusión general de carácter metodológico, se resumen a continuación los principales hallazgos particulares.

a) Los resultados del léxico disponible, que confirman el carácter de transición de las hablas castellano-manchegas a través de los materiales léxicos, permiten la elaboración de distribuciones geográficas o mapas esquemáticos, dado el carácter extensivo de la muestra.

\footnotetext{
51 José Luis Calero López de Ayala, Vocabulario..., op. cit.
} 
b) Las similitudes encontradas en la documentación de términos en el ALeCMan y las encuestas de disponibilidad muestran la estabilidad (o el cambio) de ciertas isoglosas léxicas de la comunidad, especialmente teniendo en cuenta que los informantes de las encuestas de léxico disponible son jóvenes y que el tipo de encuestas aplicadas no tiene carácter específicamente dialectal, sino que recoge palabras de uso común y frecuente. Este aspecto supone añadir una valoración diastrática a materiales de índole diatópica, lo que contribuye al estudio de la variación léxica desde una «dialectología social».

c) Los materiales del léxico disponible permiten, además de comprobar la extensión geográfica de términos aislados, identificar grupos de palabras de carácter sinonímico con una clara especialización diatópica y una estructura de matriz dialectal. Igualmente, facilita la observación de variantes morfológicas y fonéticas a través del estudio de las elecciones gráficas realizadas por los informantes.

d) Los términos dialectales que se documentan en los listados de léxico disponible permiten tanto el estudio de la variación diastrática como el estudio de la variación diatópica. En Castilla-La Mancha resulta significativa estadísticamente la división en seis zonas dialectales (La Mancha, el oeste, el sureste, el este, el noroeste y el noreste) incluso cuando se controla la posible influencia de otras variables independientes como el tipo de centro (público y privado) o el tipo de municipio (rural o urbano).

e) Teniendo en cuenta los conceptos de vitalidad y extensión, destaca por su alto grado de dialectalidad la zona sur de Albacete, mientras que el oeste de Guadalajara (área de influencia madrileña) obtiene los índices más bajos. La Mancha emerge como una de las comarcas con mayor extensión de los términos dialectales, pero de vitalidad relativa, mientras que el este de Cuenca y Albacete posee una amplitud modesta pero una vitalidad emergente. Aparece así una franja centro-este-sureste más productiva en cuanto al material dialectal disponible. Por otro lado, desde el punto de vista cualitativo, parece que existe una continuidad léxica entre la zona manchega central y el oeste de Castilla-La Mancha.

Para terminar, hay que tener en cuenta que estas afirmaciones son solo tendencias observadas a través de los materiales léxicos que ofrece la disponibilidad, que no dejan de constituir una parte limitada del léxico completo de una comunidad de habla. Aun así, en las propuestas presentadas quedan patentes las posibilidades de análisis diatópico del léxico disponible, en las cada vez más fecundas aplicaciones de esta línea de investigación. 